\title{
Research on speed and distance measurement algorithm based on multi-sensor information fusion
}

\author{
Ying Lin ${ }^{1,}$ a , Daomin Wang ${ }^{2, b}$ and Wenbin Zhang ${ }^{2, b}$ \\ ${ }^{1}$ Beijing Hollysys Co., Ltd, Beijing, 100176, China \\ ${ }^{2}$ Beijing MTR Construction Administration Corporation, Beijing, 100068, China \\ alinying19830130@163.com, ${ }^{b}$ dmwang@sina.com, 'wbzhang@sina.com
}

\begin{abstract}
Keywords: railway signal; speed and distance measurement; wheel speed sensor; acceleration sensor; information fusion

Abstract. The speed and distance measurement algorithm which is focus on experts and scholars in the field of railway signal is one of the core technology of VOBC (Vehicle On Board Controller), and many research results was born. Due to the practical application environments, equipment cost and some other issues, the current field of railway signals mainly uses the wheel speed sensor, radar speed sensor to measure the distance and speed. However, in practical applications, the problems such as the instability of speed measurement, sensor failure often lead to the impact of normal railway transportation. Aiming at the problem of speed and distance measurement algorithm, this paper proposes a speed and distance measurement algorithm based on multi-sensor information fusion of wheel speed sensor and acceleration sensor. Because the wheel speed sensor is accurate when the wheel is not slip, and the acceleration sensor is not affected by slip of the wheel, this algorithm uses this two type of sensors to judge whether the wheel is slip, and uses different information fusion methods to calculate the wheel speed and train speed by the wheel state. By the field test, this algorithm can effectively detects whether wheel is slip, and calculates the actual speed of the train, which has practical application value.
\end{abstract}

\section{Introduction}

The speed and distance measurement is one of the core technology of VOBC, which is the basis of ATC (Automatic Train Control), so it has been concerned greatly by experts in the fields of railway signal. Italian scholar Monica proposed a relevant algorithm based on wheel speed sensors in $2001^{[1,2]}$. But the wheel speed and train speed are quite different when the wheel is slip, the train speed and distance can be only calculated by the empirical parameters. The practical application proved that the computational accuracy of algorithm is very dependent on the algorithm configuration parameters which can't cover all the conditions. Therefore, the computational accuracy of algorithm is unstable when the wheel is slip. To solve this problem, the scholars have studied the speed and distance measurement algorithm, which is based on the wheel speed sensors and combined with other sensors that are not affected by slip. The representative sensors includes radar speed sensor, GPS system, inertial navigation systems, and so on. In the laboratory environment, these algorithms have a good effect on solving this problems ${ }^{[3,4,5,6,7]}$. However, radar speed sensor has higher requirement of installation and application environment, and also has higher failure rate in some environments. The GPS system is limited to the geographical environment, and it's difficult to solve the application problems of underground and tunnel environments. And the cost of inertial navigation system is too high to be engineering application.

Since the last century $60^{\text {th }}$, acceleration sensor has developed into a mature technology. The acceleration sensor which is also not affect by slip, can be installed in the train to measure the acceleration of the train. Thus it has a good working environment, and also has many advantages, such as high precision, high reliability, low cost, and convenient engineering application. In this paper, a multi-sensor information fusion algorithm based on wheel speed sensor and acceleration sensor is proposed. This algorithm combines the advantages of the two sensors and uses different information fusion algorithm to calculate the speed and distance for different situations. 


\section{Wheel state and train speed model}

According to the research results of vehicle engineering ${ }^{[8,9]}$, due to the creep principle of wheel and rail, the train speed and wheel speed are not exactly the same when the train is moving. The relationship between wheel speed and train speed can be expressed by the creep rate $\lambda$, and the equation is:

$$
\lambda=\left|\frac{V_{\text {wheel }}-V_{\text {train }}}{V_{\text {train }}}\right|
$$

Where, $V_{\text {wheel }}$ is wheel speed, $V_{\text {train }}$ is train speed.

When the train is in coast state, the wheels are rolling state, the wheel speed is equal to the train speed. When the wheel is affected by a small traction or braking force, the wheel will appear creep phenomenon, and the creep rate is small. If the traction or braking force increases, the creep rate will gradually increase too. When the force increases beyond the limit, the creep rate will quickly become larger, and the slip happens. The relationship between the creep rate and the traction or braking force is shown in Fig. 1.

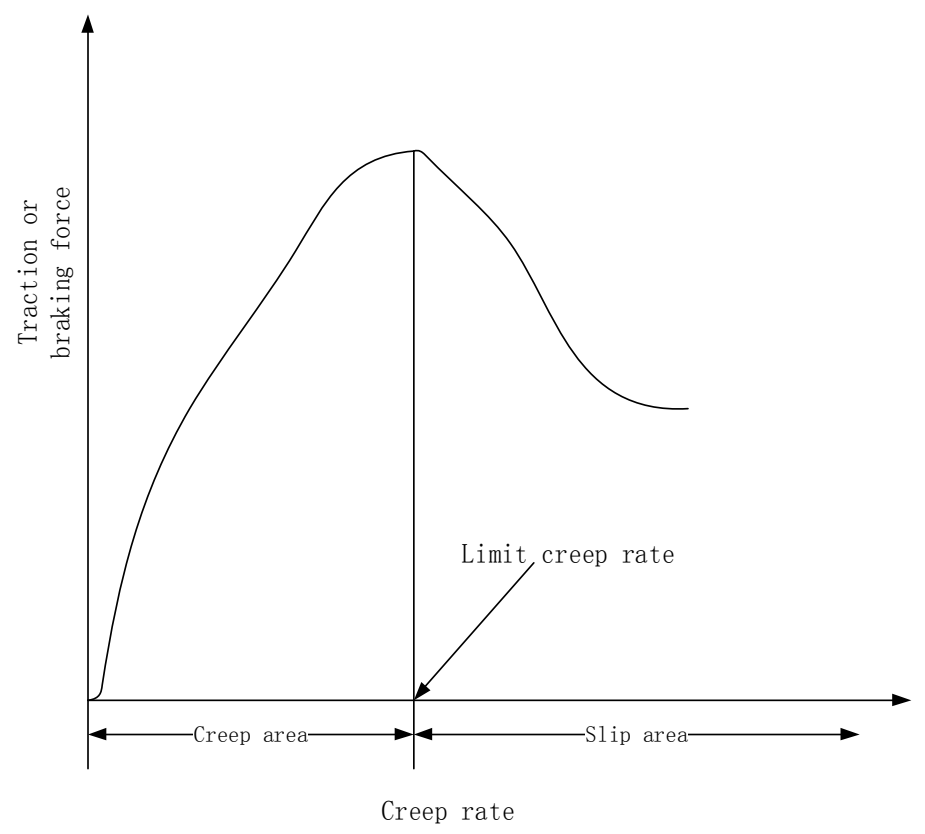

Fig. 1 The relationship between the creep rate and the traction or braking force

Fig. 1 shows, when the force is applied on the wheel, the creep rate is not equal to 0 . It means the wheel speed is not equal to the train speed. The wheel speed is greater than the train speed when train is in traction situation, and the wheel speed is less than train speed when train is in braking situation ${ }^{[8,9]}$. Therefore, speed calculation might be different error if the algorithm only uses the wheel speed as the train speed in any situation.

\section{Speed and distance measurement algorithm}

\section{Algorithm principle description}

The speed and distance measurement algorithm based on multi-sensor information fusion uses two wheel sensors and two acceleration sensors for information acquisition. The calculation principle of algorithm is shown in Fig. 2. 


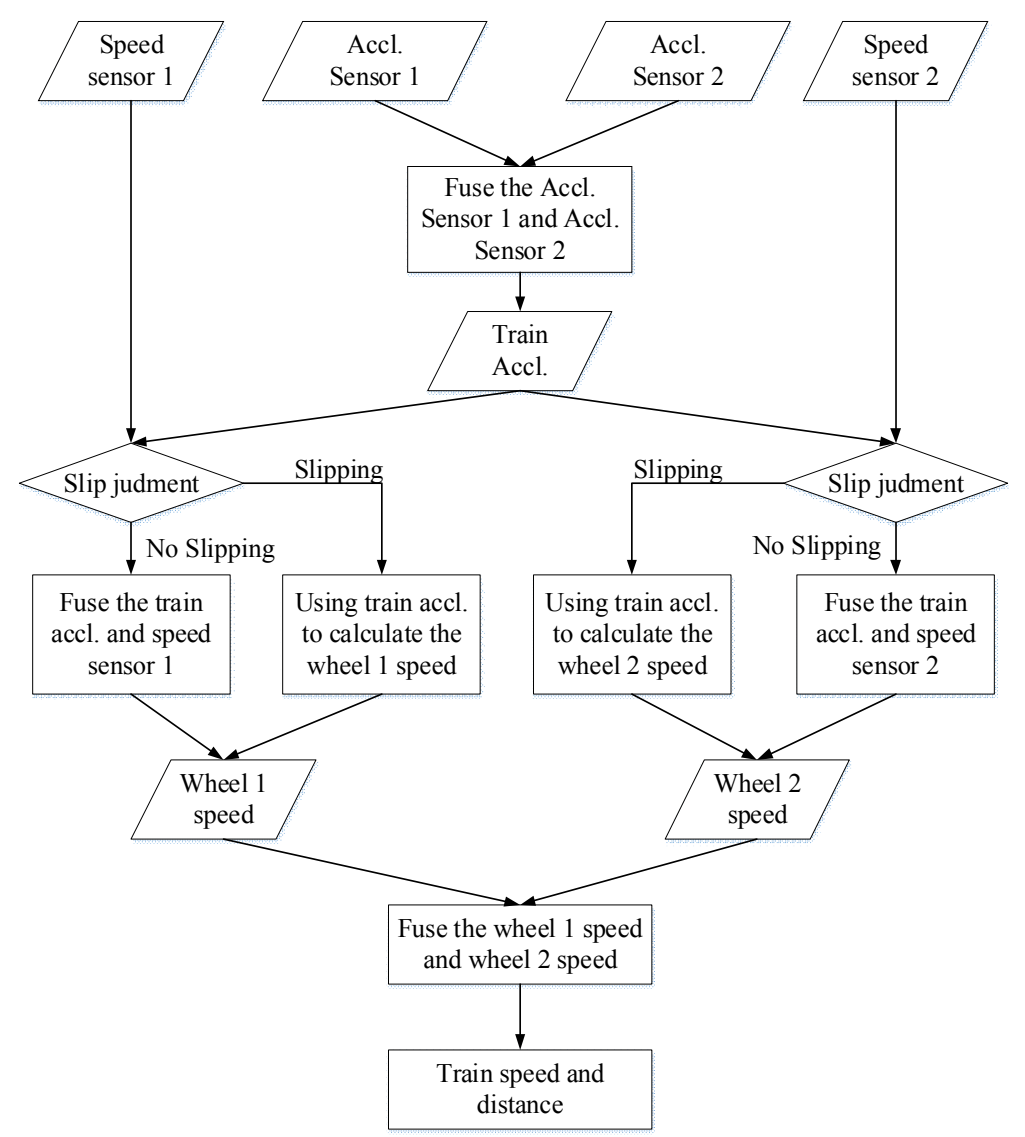

Fig. 2 The principle of speed and distance measurement algorithm

According to Fig.2, this algorithm could be described as follow: Firstly, the algorithm fuses the raw information collected by the two acceleration sensors. Secondly, by the speed information collected by the wheel sensors and information from acceleration sensors, the algorithm judges whether the wheel is slip. Thirdly, according to the wheel's state, algorithm uses different information fusion methods to calculate the speed of the two wheels. Finally, the speed and distance of the train can be calculated by the state and speed of the two wheels.

\section{Speed and distance calculation model}

Suppose the measured value of the wheel speed sensor 1 is $V_{\text {raw } 1}$, the measured value of the wheel speed sensor 2 is $V_{\text {raw_2 }}$, the measured value of acceleration sensor 1 is $A_{\text {raw } 1}$, the measured value of acceleration sensor 2 is $A_{\text {raw_2 } 2}$, and the sampling time is $T_{\text {sample }}$.

\section{Data preprocessing}

\section{(1) Train acceleration calculation model}

According to the acceleration sensor 1 and 2, calculate the train acceleration $A_{\text {meas }(i)}$ :

$$
A_{\text {meas }(i)}=\frac{A_{\text {raw_1 } 1(i)}+A_{\text {raw_2(i) }}}{2}
$$

\section{(2) Wheel acceleration calculation model}

The measured values of wheel speed sensor are not smooth. If they are directly used to calculate the wheel acceleration, the calculated wheel acceleration might be smaller or larger, even be positive or negative alternately. So, it need to smooth the raw speed of the wheel speed sensor to get the filtered speed $V_{\text {wheel_ } x(i)}$.

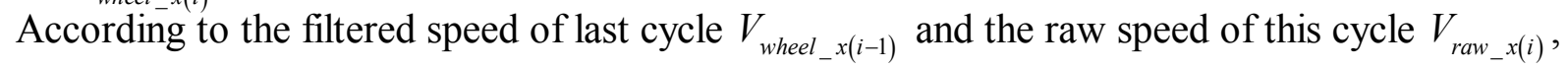
calculate the filtered speed of this cycle $V_{\text {wheel_} \_x(i)}$ : 


$$
V_{\text {wheel_x } x(i)}=\left(1-\frac{1}{p}\right) \cdot V_{\text {wheel_} \_(i-1)}+\frac{1}{p} \cdot V_{r a w_{-} x(i)}
$$

Where, $p$ is the filter parameters, the value is greater, the speed calculation delay is greater.

By the filtered speed of last cycle and the filtered speed of this cycle, calculates the wheel acceleration of this cycle $A_{\text {wheel_t } x(i)}$ :

$$
A_{\text {wheel_x }(i)}=\frac{V_{\text {wheel_x }(i)}-V_{\text {wheel_x }(i-1)}}{T_{\text {sample }}}
$$

Where, $V_{\text {wheel_ } \mathrm{x}(i-1)}$ is the $\mathrm{x}_{\mathrm{th}}$ wheel's filtered speed of $(\mathrm{i}-1)_{\text {th }}$ cycle.

\section{Slip judgement model}

The algorithm uses the acceleration detection method and the speed difference detection method to detect the state of the wheel, and judge whether the wheel is slip. Then determine the specific state of wheel by the wheel state transition model.

\section{(1) Acceleration detection method}

The basic principle of the acceleration detection method is that if the wheel is not slip, the wheel acceleration calculated by the wheel speed sensor and the train acceleration calculated by acceleration sensor should be consistent.

According to the wheel acceleration $A_{\text {wheel_x(i) }}$ and train acceleration $A_{\text {meas }(i)}$ of this cycle, calculate the difference $A_{d i f_{-} x(i)}$ of this cycle:

$$
A_{\text {dif_c } x(i)}=A_{\text {meas }}-A_{\text {wheel_} \_(i)}
$$

When the train is operating, especially in the case of overload, the impulsion of train might be bigger in both traction and barking situation. Thus, the measured value collected by acceleration sensor may be saltation and it may lead to misjudging. Therefore, the calculation of the Eq. 5 needs to be smoothed to prevent misjudgment.

Based on Eq. 5, combining the difference between the wheel and train acceleration of last cycle, calculates the difference $A_{d i f_{-} x(i)}$ of this cycle:

$$
A_{\text {dif_x } x(i)}=\left(1-\frac{1}{p}\right) \cdot A_{\text {dif_- } x(i)}+\frac{1}{p}\left(A_{\text {meas }}-A_{\text {wheel_ } x(i)}\right)
$$

According to the calculation result of Eq. 6, if $\left|A_{d i f_{-} x(i)}\right| \geq A c c_{\text {slip }}$, it means that the acceleration detection method find the $\mathrm{i}_{\text {th }}$ wheel is slip, and the flag of this detection method $A_{\text {slip }}$ should be set as true. On the contrary, no slip is detected. $A c c_{\text {slip }}$ is the threshold of acceleration detection method for slip judgement. The value is greater, the detection is looser, and the value is smaller, the detection is stricter.

\section{(2) Velocity difference detection}

The basic principle of the speed difference detection method is that if the wheel is not slip, the wheel speed calculated by the acceleration sensor and wheel speed sensor should be consistent.

Based on the calculated acceleration of train by Eq. 2, calculate the wheel speed $V_{\text {temp } \_x(i)}$ :

$$
V_{\text {temp_x(i) }}=V_{\text {calculated_x(i) }}+A_{\text {meas }} \cdot T_{\text {sample }}
$$

By the wheel speed $V_{\text {wheel_x(i) }}$ calculated by wheel speed sensor and the wheel speed

$$
V_{\text {temp_ } x(i)} \text { calculated by acceleration sensor, calculates their difference } V_{d i f_{-} x(i)} \text { : }
$$

$$
V_{\text {dif_- } x(i)}=V_{\text {temp_x } x(i)}-V_{\text {wheel_x }} x(i)
$$


According to the calculation result of Eq. 8, if $\left|V_{d i f_{-} x(i)}\right| \geq V e l_{\text {slip }}$, it means that the speed difference detection method find the $\mathrm{i}_{\text {th }}$ wheel is slip, and the flag of this detection method $V_{\text {slip }}$ should be set as true. On the contrary, no slip is detected. $V e l_{\text {slip }}$ is the threshold of this detection method for slip judgement. The value is greater, the detection is looser, and the value is smaller, the detection is stricter.

\section{(3) Wheel state transition model}

The algorithm can detect whether the wheel is slip by the acceleration detection method and the speed difference detection method, but be unable to determine whether the wheel is slide or spin. At the same time, the intermediate state due to the filter delay must be described, such as the wheel returns normal state from slide or spin.

The algorithm divides the wheel state into 4 types which are normal state, slide state, spin state and implausible state. The state transition model is shown in Fig. 3.

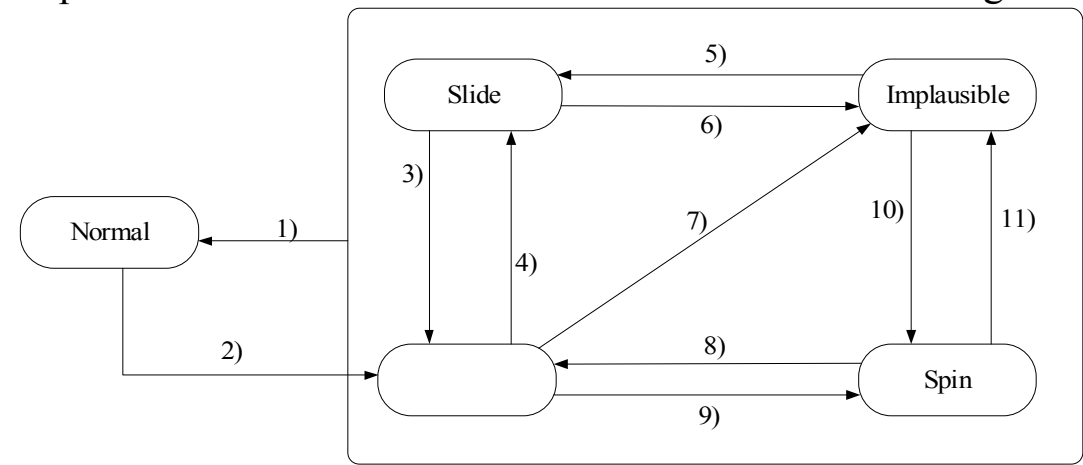

Fig. 3 The state transition model of wheel

The state transition conditions in Fig. 3 are as follows:

1) $\mathrm{V}=0$ or $\mathrm{A}_{\text {slip }}=$ false and $\mathrm{V}_{\text {slip }}=$ false;

2) $\mathrm{V}>0$ or $\mathrm{A}_{\text {slip }}=$ true and $\mathrm{V}_{\text {slip }}=$ true;

3) $A_{\text {meas }} \geq$ SLIDE_PLAUSIBLE_MAX;

4) $V_{\text {slip }}=$ true and $V_{\text {dif }}<0$ and $A_{\text {meas }}<$ SLIDE_PLAUSIBLE_MAX;

5) $\mathrm{V}_{\text {dif }}<0$ and $\mathrm{A}_{\text {meas }}<$ SLIDE_PLAUSIBLE_MAX and $\mathrm{A}_{\text {meas }}<=$ SPIN_PLAUSIBLE_MIN;

6) $V_{\text {dif }}>0$ and $A_{\text {meas }}<$ SLIDE_PLAUSIBLE_MAX

7) ( $\mathrm{V}_{\text {slip }}=$ true and $\mathrm{V}_{\text {dif }}<0$ and $\mathrm{A}_{\text {meas }}>=\mathrm{SLIDE}$ _PLAUSIBLE_MAX) or $\left(\mathrm{V}_{\text {slip }}=\right.$ true and $\mathrm{V}_{\text {dif }}>=$ 0 and $\mathrm{A}_{\text {meas }}<=$ SPIN_PLAUSIBLEM_NIN)

8) $\mathrm{A}_{\text {meas }}<=$ SPIN_PLAUSIBLE_MIN

9) $\mathrm{V}_{\text {slip }}=$ true and $\mathrm{V}_{\text {dif }}>=0$ and $\mathrm{A}_{\text {meas }}>$ SPIN_PLAUSIBLE_MIN

10) $V_{\text {dif }}>=0$ and $A_{\text {meas }}>$ SLIDE_PLAUSIBLE_MAX and $A_{\text {meas }}>$ SPIN_PLAUSIBLE_MIN

11) $\mathrm{V}_{\text {dif }}<0$ and $A_{\text {meas }}>$ SPIN_PLAUSIBLE_MIN

Where, SLIDE_PLAUSIBLE_MAX and SPIN_PLAUSIBLE_MIN are the parameters for state machine.

\section{Train speed calculation model}

Train speed calculation model first calculates the two wheels' speed separately, and then calculates the train speed.

(1) Wheel speed calculation model

According to algorithm, the wheel state is divided into normal state, slide state, spin state and implausible state. Only wheel is in normal state, the wheel speed and train speed are similar and the wheel speed and train speed can be calculated by wheel speed sensors and acceleration sensors. In the 
other three states, the wheel speed and train speed are quite different and the wheel speed and train speed can be only calculated by acceleration sensors.

\section{$>$ Wheel speed calculation model for normal state}

In the second part of this paper, it has been described that due to the creep rate, the calculation speed of train will be larger or lower if algorithm only uses the wheel speed as train speed directly. In order to solve this problem, algorithm fuses the wheel speed calculated by wheel sensors and the wheel speed calculated by acceleration sensors to calculate the final wheel speed.

According the calculation result of Eq. 3 and Eq. 7, calculates the final wheel speed $V_{\text {calculated_x }_{-}(i)}$ :

$$
V_{\text {calculated_ } \_(i)}=\left(1-\frac{1}{p}\right) \cdot V_{\text {temp_- } x(i)}+\frac{1}{p} \cdot V_{\text {wheel_x } \_(i)}
$$

Where, $p$ is the information fusion weight of sensors.

It can be known in the description of second part of this paper, the creep rate increases as traction or braking force increases. So, this paper calculates the max adhesion by the adhesive characteristic curve and the current speed of the train. According to Fig. 1, when the adhesion is equal to 0, the creep rate is 0 , the weight of sensor data is taken as 1 . When the adhesion is the biggest, calculate the max limit creep rate as $\lambda_{\max }$, and the weight of sensor data is taken as infinity. Then using primary hyperbola fitting method to get the relationship equation between adhesion and the information fusion weight of sensors. Finally, substituting the current train force, which calculated by acceleration sensor, into the correlation relationship equation to calculate the data fusion weight of sensor data.

According to the calculation result of Eq. 2 and train's weight $M$, calculates the current train force $F_{(i)}$ :

$$
F_{(i)}=M \cdot A_{\text {meas }(i)}
$$

According adhesive characteristic curve, calculate the max adhesion $F_{\max }$ for current speed. Make a primary hyperbola fit under these two conditions, one is that the adhesion is $0, p$ is 1 , and the other condition is that the adhesion is equal to $F_{\max }$ and $p$ is infinity. The following equation can be obtained.

$$
p=\frac{a}{F+a}
$$

Finally, substituting the train force $F_{(i)}$ into Eq. 11, calculates the data fusion weight of sensor data $p$.

\section{$>$ Wheel speed calculation model for other state}

When the wheel is not in normal state, the wheel speed which is calculated by wheel sensor is not accurate. So the wheel speed should be calculated by acceleration sensor as the final speed which is the result of Eq. 7.

(2) Train speed calculation model

Combining the two wheel states can be obtained in the following three situations:

1) Both wheels are in normal state;

2) One wheel is in normal state and the other one is in other states.

3) Both wheels are in other states.

\section{Both wheels are in normal state}

The wheel speed calculated by wheel speed sensor and acceleration sensors is accurate. So when the two wheels are in normal state, the train speed $V_{\text {train }}$ is calculated by taking the average value of two wheel speed:

$$
V_{\text {train }(i)}=\frac{V_{\text {calculated_1(i) }}+V_{\text {calculated_2(i) }}}{2}
$$




\section{$>$ One wheel is in normal state and the other one is in other states.}

In this condition, algorithm uses the wheel speed which wheel is in normal state as the train speed $V_{\text {train }}$ :

$$
V_{\text {train }(i)}=V_{\text {calculated_1(i) }} \quad \text { or } \quad V_{\text {calculated_2(i) }}
$$

\section{$>$ Both wheels are in other states}

In this condition, the train speed $V_{\text {train }}$ is calculated by taking the average value of two wheel speed which is calculated by acceleration sensors. The equation is as follows:

$$
V_{\text {train }(i)}=\frac{V_{\text {calculated_1(i) }}+V_{\text {calculated_2(i) }}}{2}
$$

\section{Algorithm verification and analysis}

In order to verify the correctness of the algorithm, this paper makes field tests on the test railway in Tongji University. The test vehicle is A type of urban rail transit, and mode of train formation is 1M1T. Two speed sensors which is DF-16 type of 200 teeth, are installed in different brake axles. Two acceleration sensors which is LSOX-LC-0.5G-5264 type, are installed in the train. The sampling period is 100 s. At the same time, a DRS05 radar speed sensor is installed on the train as a reference of actual train speed to verify the accuracy of the algorithm.

For the purpose of verifying the algorithm in all situation, the field test includes single wheel slide tests, two wheels slide tests, and different speed grade tests. The test results are shown in Fig. 4, Fig. 5, Fig. 6 and Table 1.

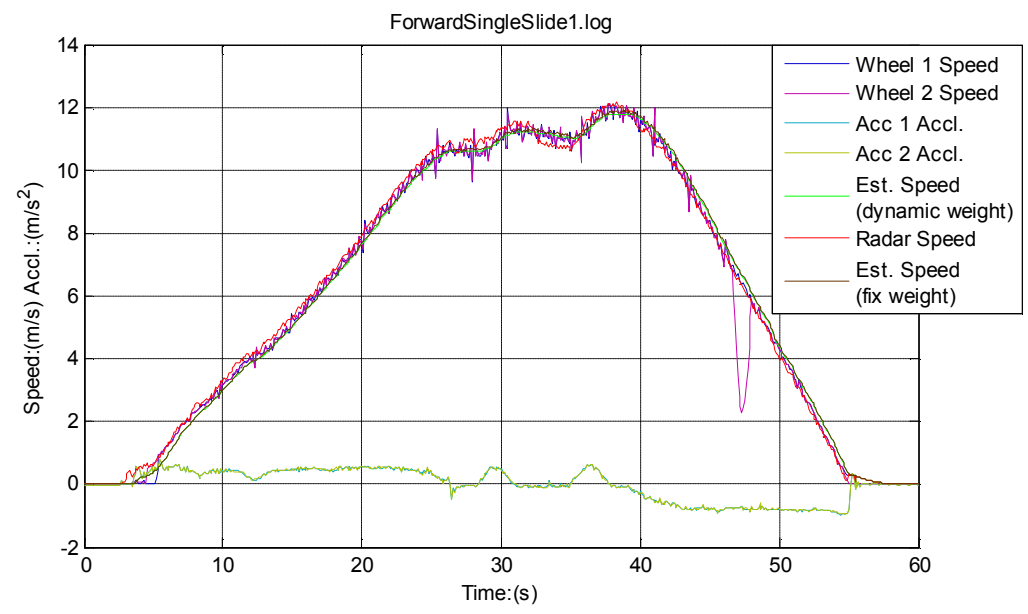

Fig. 4 Single wheel slide test result

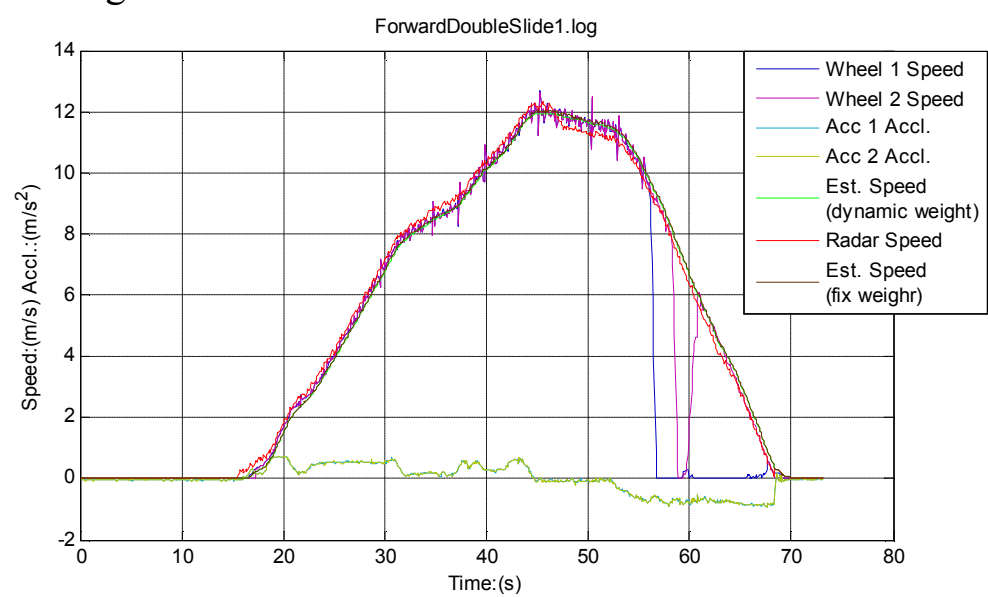

Fig. 5 Double wheel slide test result 


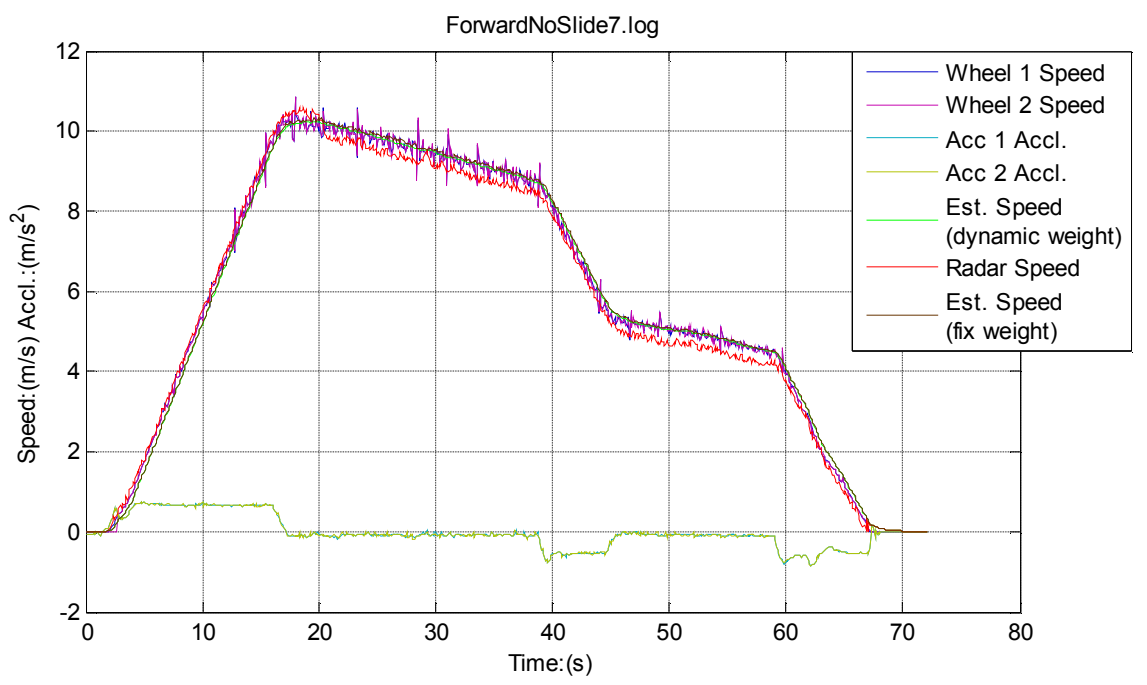

Fig. 6 No slip test result

Table 1 The test results (partial data)

\begin{tabular}{|c|c|c|c|c|}
\hline $\begin{array}{c}\text { Test } \\
\text { sequence }\end{array}$ & $\begin{array}{c}\text { the max error } \\
\text { between dynamic } \\
\text { weight algorithm } \\
\text { and radar velocity } \\
\text { measurement } \\
{[\mathrm{km} / \mathrm{h}]}\end{array}$ & $\begin{array}{c}\text { the average error } \\
\text { between dynamic } \\
\text { weight algorithm } \\
\text { and radar velocity } \\
\text { measurement } \\
{[\mathrm{km} / \mathrm{h}]}\end{array}$ & $\begin{array}{c}\text { the max error } \\
\text { between Fixed } \\
\text { weight algorithm } \\
\text { and radar velocity } \\
\text { measurement } \\
{[\mathrm{km} / \mathrm{h}]}\end{array}$ & $\begin{array}{c}\text { the average error } \\
\text { between Fixed } \\
\text { weight algorithm } \\
\text { and radar velocity } \\
\text { measurement } \\
{[\mathrm{km} / \mathrm{h}]}\end{array}$ \\
\hline 1 & 1.37 & 1.09 & 2.00 & 1.67 \\
\hline 2 & 1.32 & 0.88 & 1.67 & 1.45 \\
\hline 3 & 1.12 & 0.92 & 1.92 & 1.69 \\
\hline 5 & 1.09 & 0.96 & 1.82 & 1.72 \\
\hline 6 & 0.96 & 0.81 & 1.77 & 1.52 \\
\hline 7 & 1.01 & 0.94 & 1.69 & 1.55 \\
\hline
\end{tabular}

According to the Fig. 4, Fig. 5, Fig. 6 and Table 1, they can be shown that the estimated train speed calculated by the algorithm is smooth under different situations. And there is no speed jump, and the speed error between the train speed calculated by the algorithm and the radar speed sensor is always not more than $1.5 \mathrm{~km} / \mathrm{h}$ which meets the requirement of IEEE $1474.1^{[10]}$. In addition, when the data fusion weight of sensor data is set as fixed value, the speed detection error is significantly greater than the dynamic value. It means that adjust the data fusion weight of sensor data by creep rate can effectively improve the accuracy of the calculation speed, when the wheels are no slip.

\section{Conclusions}

The speed and distance measurement algorithm is one of the core technology of VOBC, which directly affects the automatic speed protection and automatic speed operation function. Aiming at the problems existing in the mainstream algorithm, a speed and distance measurement algorithm based on multi-sensor information fusion is proposed by this paper. Based on wheel state and train speed model, the algorithm dynamically adjusts the data fusion weight of sensor data to fuse the data of wheel speed sensor and acceleration sensor and improves the accuracy of the calculation speed. According to the results of filed tests, they are proved that the algorithm can effectively detect the whether the wheel is slip, and uses different information fusion methods to calculate train speed by wheel's state. The calculation train speed is smooth and accuracy of calculation speed reaches the requirement of IEEE $1474.1^{[10]}$. By adding acceleration sensors, the algorithm proposed in this paper has the advantages, such as convenient installation, convenient maintenance, high measuring accuracy, low cost, and so on. 
So it can meet the requirements of engineering application and have practical application value for VOBC.

\section{Reference}

[1] T.P.M. Monica, A. Benedetto, C. Valentina. Train Speed and Position Evaluation using Wheel Velocity Measure. IEEE/ASME International Conference on Advanced Intelligent Mechatronics Proeeedings.Italy.2001, p. 117-121

[2] A. Benedetto, C. Valentina, M. Monica. Train Position and Speed Estimation Using Wheel velocity measurement. Proceedings of the IMech E, Part F, Journal of Rail and Rapid Transit, 2002, p. 216

[3] Lin Ying, Wang Changlin. Research of slip and slide detection and adjust method in onboard ATP. Urban Mass Transit, Vol. 3(2011), p. 24-27. "In Chinese"

[4] Zhou Datian, Tang Tao, Wang Shaowei, Guo Baoqing. Train locating by time-domain correlation and matching based on information fusion. Journal of China railway society. Vol. 28(2006), p. 97-100. "In Chinese"

[5] Qiao Chao, Tang Huijia. The method of positioning trains by odometers. Journal of Lanzhou railway university (natural sciences). Vol. 22(2003), p. 66-71. "In Chinese"

[6] Tang Wei, Sun Zhifang, Chen Quan. On board DPS-Based radar speed measurement system. Techniques of automation and application, Vol. 25(2006), p. 20-22. "In Chinese"

[7] Liu Yue, Ning Bin. Exploration of train speed and distance measurement. Railway Signalling \& Communication. Vol. 33(1997), p. 1-3. "In Chinese"

[8] Xiao Qian, Wang Chengguo, Zhou Xinjian, Lu Yong. Analysis on the characteristics of wheel/rail rolling contact under different friction coefficient. China railway science. Vol. 32(2011), p. 66-71. "In Chinese"

[9] Ni Pingtao, Wang Kaiwen, Zhang Weihua, Chi Maoru. Calculation method of wheel -rail contact relation. Journal of traffic and transportation engineering. Vol. 6(2006), p. 10-13. "In Chinese"

[10] Rail Transit Vehicle Interface Standards Committee of the IEEE Vehicular Technology Society . IEEE 1474.1TM IEEE Standard for Communications-Based Train Control (CBTC) Performance and Functional Requirements. The United States of America: the Institute of Electrical and Electronics Engineers, Inc. (2005). 